\title{
Etude de la qualité des eaux consommées dans la commune d'Adjohoun au Bénin
}

\author{
Elisabeth YEHOUENOU A PAZOU ${ }^{1 *}$, David AZOCLI ${ }^{1}$, Antoine Vikkey HINSON ${ }^{2}$, \\ Bénoîte ASSOGBA ${ }^{1}$, Hermine AVOCE ${ }^{1}$ et Elodie HOZANHEKPON ${ }^{1}$
}

\author{
${ }^{1}$ Laboratoire de Recherche en Biologie Appliquée (LARBA), Département de Génie de l'Environnement, \\ Ecole Polytechnique d'Abomey-Calavi, Université d'Abomey-Calavi, Cotonou, 01 BP 2009, Bénin YAPE : \\ yehouenou@yahoo.fr; Tél. : +22995841807. \\ ${ }^{2}$ Unité d'Enseignement et de Recherche de Santé au Travail et Environnement, Faculté des Sciences de la Santé \\ Cotonou, Bénin. \\ *Auteur correspondant ; E-mail: yehouenoue@yahoo.fr
}

\section{RESUME}

La présente étude porte sur la qualité de l'eau consommée dans 7 villages de la commune d'Adjohoun. Les différents entretiens avec la population ont révélé que l'eau consommée dans cette localité a toujours été source de graves maladies telles que : la diarrhée, la bilharziose, le choléra. Des analyses physico - chimiques comme le $\mathrm{pH}$, la conductivité, la couleur, le calcium, le chlore ont été effectués avec un multiparamètre de marque COMBO BY HANNA et la recherche des coliformes totaux, des coliformes fécaux et des streptocoques fécaux sont effectuées par les méthodes d'ensemencement sur milieux de culture. Les résultats des analyses physicochimiques ont révélé que la conductivité varie entre 20 et $64 \mu \mathrm{s} / \mathrm{cm}$; le pH se situe entre 4,77 et 6,55. La couleur très élevée varie entre 57 et $226 \mathrm{UC}$, le chlore entre $10,65 \mathrm{mg} / \mathrm{l}$ et $320 \mathrm{mg} / \mathrm{l}$; le calcium entre 0,81 et $8,817 \mathrm{mg} / \mathrm{l}$. L'analyse bactériologique des eaux révèle que le taux de coliformes totaux $(17600 ; 14400 ; 22320 ; 3500$ UFC $/ 100 \mathrm{ml})$, de coliformes fécaux $(1000 ; 1200 ; 3200 ; 250$ UFC /100 ml), et de streptocoques fécaux $(600 ; 660$; 900 ; 200 UFC /100 ml) ne sont pas conformes aux normes d'une eau de boisson. Cette contamination est d'origine fécale et s'explique par les nombreux cas de maladies hydriques enregistrées.

(C) 2018 International Formulae Group. All rights reserved.

Mots clés: Qualité, eau de consommation, commune d'Adjohoun, Bénin

\begin{abstract}
This study examines water quality consumed in seven municipalities of Adjohoun. Various interviews with population revealed that water consumed has always been the cases of serious diseases such as: diarrhea, bilharzia, cholera in these localities. Physico - chemical analysis such as $\mathrm{pH}$, conductivity, la color, calcium, chloride were done with Hanna multiparameter. Bactériological analysis such as total coliforms, fecal coliforms and fecal streptocoques fécaux were also done. Results shown that conductivity varied from 20 to $64 \mu \mathrm{s} / \mathrm{cm} ; \mathrm{pH}$ was between 4.77 and 6.55. Water color was very high and varied from 57 to 226 US. Chlorine was between $10.65 \mathrm{mg} / 1$ and $320 \mathrm{mg} / 1$; calcium between 0.81 and $8.817 \mathrm{mg} / 1$. Bacteriological analysis of the water revealed that total coliforms $(17600,14400,22320,3500 \mathrm{UFC} / 100 \mathrm{ml})$, faecal coliforms $(1000,1200,3200,250$ UFC $/ 100 \mathrm{ml})$ and faecal streptococci $(600 ; 660 ; 900$; are higher than standards drinking water. These fecal contamination could explain numerous diseases registered in theses localities.
\end{abstract}

(C) 2018 International Formulae Group. All rights reserved.

Keywords: Consumed water quality, Adjohoun, Benin. 


\section{INTRODUCTION}

L'eau, ressource d'intérêt à multiples fonctions est un composant essentiel des êtres vivants puisque l'eau contribue au bon fonctionnement et à l'équilibre de la physiologie humaine. La survie de l'homme exige donc une eau saine et en quantité suffisante ASSITEB, 1997. Le manque d'eau a amené, en mars 1977, la Communauté Internationale à se réunir à Mar Del Plata en Argentine, pour décréter la période 1981-1990 comme la Décennie Internationale de l'Eau Potable et de l'Assainissement (DIEPA). L'objectif fixé par cette conférence pour l'année 1990 a été d'assurer à l'ensemble de la population mondiale, l'accès à l'eau potable et aux services d'assainissement afin de diminuer les maladies hydriques (OMS, 1990).

Vu l'importance de l'eau pour la santé, la survie, la croissance et le développement, la communauté mondiale s'est engagée dans l'Objectif $\mathrm{n}^{\circ} 7$ du Millénaire pour le Développement (OMD) à réduire de moitié le nombre de personnes n'ayant pas accès de façon durable à l'eau de boisson et à l'assainissement de base d'ici 2015 (OMS/UNICEF, 2007). Malheureusement, l'accès à l'eau potable constitue toujours un véritable problème dans les pays en voie de développement, surtout ceux africains. C'est le cas de l'Afrique sub-saharienne qui reste la région où la situation demeure la plus préoccupante. En effet, entre 1990 et 2004, le nombre de personnes n'ayant pas accès à l'eau potable y a augmenté de $23 \%$, celles privées d'assainissement de plus de $30 \%$ (OMS/UNICEF, 2007).

Au Bénin, la question de l'eau constitue un problème majeur pour la population pourtant le Bénin dispose d'un important potentiel hydrique globalement satisfaisant qui peut le mettre à l'abri de toute pénurie pour ses activités de développement socio-économique. La République du Bénin reçoit les hauteurs moyennes annuelles de pluies qui varient entre 700 et $1000 \mathrm{~mm}$ dans la zone Nord et entre 1000 et $1200 \mathrm{~mm}$ dans le reste du pays
(CCPRB, 2005), mais les capacités du pays à mobiliser ces ressources pour faire face à la demande en eau potable sont encore insuffisantes (Zannou, 2006). La Société Nationale des Eaux du Bénin (SONEB) s'est occupée de l'alimentation des zones urbaines en eau de consommation et la Direction Générale de l'eau (DG Eau) de desservir les zones rurales à partir des réserves souterraines en eau. Malgré tous les efforts consentis par ces deux structures, l'atteinte des objectifs de la décennie de l'eau qui est de fournir à $80 \%$ de la population de l'eau potable à raison de 60 litres par jour à chaque habitant de la zone urbaine et 10 à 20 litres par jour à chaque habitant en milieu rural ne sont pas atteints. Face à cette situation, les populations n'hésitent pas à avoir recours aux eaux de qualité douteuse telles que les eaux de surface (fleuves, rivières, marigots, marres, lacs, lagunes) pour satisfaire leurs besoins quotidiens.

$\mathrm{Au}$ regard de ces antécédents, il est pertinent d'étudier la qualité de l'eau consommée dans les villages de Adigbonou de Koudo (deux localités de Sissekpa), de Goutonou (localité de Gbékandji), de Gla, Hlankpa, Gbanan et Gouké de la commune d'Adjohoun

\section{MATERIEL ET METHODES Zone d'étude}

La Commune d'Adjohoun (Figure 1), avec une superficie totale d'environ $308 \mathrm{~km}^{2}$ est située au centre du Département de l'Ouémé, dans la vallée et à $32 \mathrm{~km}$ de Porto Novo. Elle est limitée au Sud par la commune de Dangbo, au Nord par celle de Bonou ; à l'Est par la Commune de Sakété et à l'Ouest par les Communes d'Abomey-Calavi et de Zê (PDC Adjohoun, 2004). Administrativement, la commune d'Adjohoun est constituée de cinquante-sept (57) villages et quartiers de ville repartis dans huit (8) arrondissements à savoir : Adjohoun ; Akpadanou ; Awonou ; Azowlissè. Dème ; Gangban ; Kodé et Togbota. Sa population est d'environ 74956 habitants (RGPH4 ; INSAE, 2013). La 
population d'Adjohoun est très jeune, avec plus de $80 \%$ de personnes âgées de moins de 40 ans. Elle est essentiellement rurale et plus de $80 \%$ de la population active est occupée dans le secteur agricole. (Monographie d'Adjohoun, 2006).

\section{Critère de choix des sites de prélèvement}

Le critère de sélection des sites de prélèvement, est basé sur l'absence du réseau de la SONEB dans le village et sur l'affluence des populations vers les points d'eau ciblés.

\section{MATERIEL}

Plusieurs matériels ont été utilisés parmi lesquels on compte: un GPS (pour prendre les coordonnées géographiques des points de prélèvement); des flacons en verre de $500 \mathrm{ml}$ qui ont été soigneusement lavés, rincés à l'eau distillée, enveloppés dans du papier aluminium puis stérilisés à l'étuve à $120{ }^{\circ} \mathrm{C}$; une glacière contenant des morceaux de glace, un spectrophotomètre de type HACH DR/2800 et un multi paramètre de type COMBO BY HANNA pour le dosage des paramètres physiques et chimiques. Les différents paramètres chimiques ont été dosés avec des kits de réactifs. Des milieux de culture comme le milieu CHAPMAN TTC AGAR (TERGITOL 7 AGAR), le SALNETZ AGAR BASE.

\section{Méthodes d'Enquêtes}

Des enquêtes ont été menées dans les différents villages afin de recenser les usages que les populations font de l'eau. Les différentes analyses ont été effectuées au laboratoire de la direction Générale de l'Eau (DG Eau).

\section{Prélèvement des échantillons d'eau}

Deux campagnes de prélèvement ont été effectuées, pendant la grande saison sèche (janvier - mars) et pendant la petite saison sèche (août - septembre) où on note une forte affluence et activités humaines autour des points d'eau. Pour les analyses bactériologiques, le prélèvement des échantillons a été fait dans des flacons en verre de $500 \mathrm{ml}$. Quant aux paramètres physicochimiques les eaux ont été prélevées dans des flacons en polyéthylène de 1,51 . Sur le terrain, tous ces matériels ont été soigneusement rincés deux à trois fois au moins à l'eau à échantillonner. Ensuite, le prélèvement a été fait de manière à ce que des bulles d'air ne se forment pas dans les flacons. Les échantillons ont été ensuite conservés dans une glacière contenant de la glace et ont été transportés au laboratoire le jour où les prélèvements ont été faits. Les échantillons d'eau prélevés sont ensuite conservés dans un réfrigérateur à une température de $4{ }^{\circ} \mathrm{C}$ jusqu'au lendemain.

\section{Détermination des paramètres physiques et chimiques}

Détermination du pH, de la conductivité et de la température : ces trois paramètres ont été analysés grâce à un multi-paramètre du type HANNA dont les électrodes ont été rincées à l'eau distillée et à l'eau échantillonnée. Les valeurs obtenues ont été lues directement sur l'écran de l'appareil après avoir plongé les électrodes rincées dans l'eau échantillonnée.

Détermination de la couleur : On a prélevé 10 $\mathrm{ml}$ de l'échantillon dans un tube et $10 \mathrm{ml}$ de l'eau distillée dans un autre, on a lu le zéro avec le tube contenant l'eau distillée et ensuite la valeur de la couleur de l'échantillon sur l'écran du spectrophotomètre. La lecture a été faite à $455 \mathrm{~nm}$ avec le spectrophotomètre multi paramètre de type COMBO BY HANNA

Détermination du taux Chlorures $\left(\mathrm{Cl}^{-}\right)$: A

$100 \mathrm{ml}$ de l'échantillon à analyser, on a ajouté à l'eau prélevée, 2 gouttes de bichromate de potassium qui lui a donné une couleur jaune qu'on a dosé avec une solution de nitrate d'argent.

Détermination du taux de Bicarbonate $\left(\mathrm{HCO}_{3}{ }^{-}\right)$: A $100 \mathrm{ml}$ d'échantillon prélevé dans un erlenmeyer, on a ajouté 8 gouttes d'indicateur mixte qui lui a donné une couleur bleue. Puis on a dosé le mélange par une solution d'acide sulfurique $\left(\mathrm{H}_{2} \mathrm{SO}_{4}: 0,1 \mathrm{~N}\right)$.

Détermination du taux de Nitrate $\left(\mathrm{NO}_{3}{ }^{-}\right)$: On a prélevé dans un flacon de $25 \mathrm{ml}$ de l'échantillon d'eau à analyser auquel on a ajouté une gélule de réactif de «Nitra Ver. Dans un second flacon servant de témoin on a prélevé $25 \mathrm{ml}$ d'eau distillée puis on a ajouté la même gélule. On a agité les deux flacons pendant $1 \mathrm{mn}$, et après $5 \mathrm{~min}$ de réaction, on a fait la lecture du témoin puis celle de l'échantillon à $500 \mathrm{~nm}$. 
Détermination du taux de Nitrite $\left(\mathrm{NO}_{2}^{-}\right)$: On a prélevé $25 \mathrm{ml}$ d'eau échantillonnée dans un flacon, on y a ajouté une gélule "Nitri ver» puis on a réalisé un témoin avec $25 \mathrm{ml}$ d'eau échantillonnée sans la gélule. On a attendu 20 minutes avant de faire la lecture

Détermination du taux de sulfate ( $\left.\mathrm{SO}_{4}^{2-}\right)$ : On a prélevé $25 \mathrm{ml}$ d'eau échantillonnée dans un flacon et on y a ajouté une gélule de «sulfa ver» puis on a réalisé un blanc avec $25 \mathrm{ml}$ d'eau échantillonnée sans la gélule. On a attendu 5 minutes avant de faire la lecture.

Détermination du taux d'ammonium $\left(\mathrm{NH}_{4}{ }^{+}\right)$: On a prélevé $25 \mathrm{ml}$ d'eau échantillonnée dans un flacon; on $\mathrm{y}$ a ajouté $1 \mathrm{ml}$ de sel « Rochelle » et $1 \mathrm{ml}$ de réactif de Nessler. On a réalisé un témoin avec l'eau distillée et un $1 \mathrm{ml}$ de chaque réactif utilisé ; puis on a attendu
5 minutes pour la lecture à la longueur d'onde de 400 à $425 \mathrm{~nm}$.

\section{Analyse bactériologique}

L'analyse bactériologique a permis de déterminer la présence des coliformes totaux et fécaux, des streptocoques totaux et fécaux et des Escherichia Coli. La réalisation a été faite par les méthodes de filtration, d'ensemencement direct, et de dénombrement en utilisant les milieux de culture comme CHAPMAN TTC AGAR, SALNETZ AGAR BASE

\section{Analyse des données}

Les données ont été traitées avec les logiciels Word et Excel.

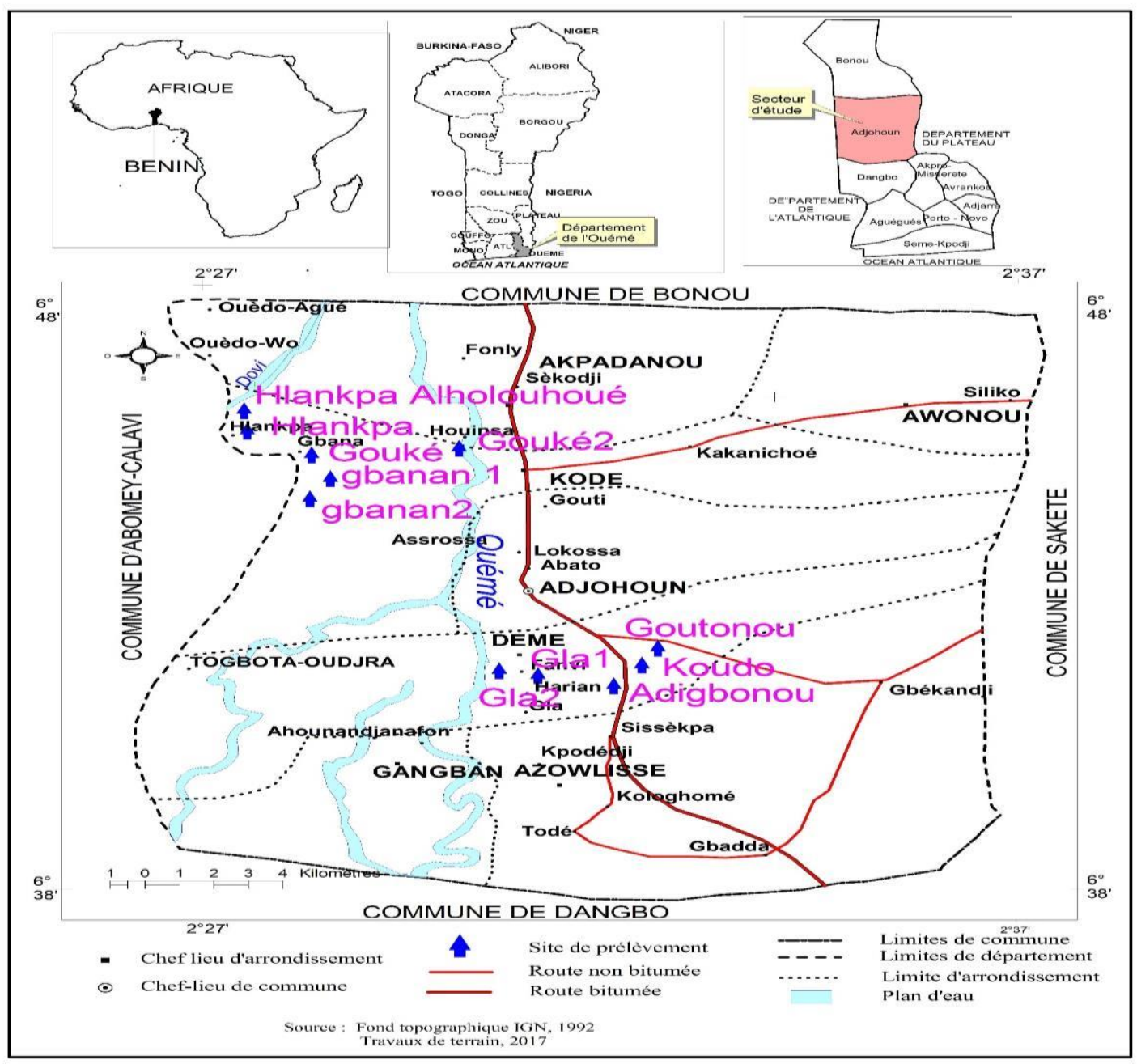

Figure 1 : Sites de prélèvement des eaux. 


\section{RESULTATS}

Il ressort des résultats des enquêtes menées dans les ménages que les populations des villages Adigbonou, Koudo (deux localités de Sissekpa), Goutonou (localité de Gbékandji). Gla et Hlankpa de la Commune d'Adjohoun n'ont pas accès à l'eau de la SONEB. L'eau de source est utilisée par la population pour faire la lessive, la vaisselle, la toilette, la cuisine et surtout comme eau de boisson. La majeure partie de la population consomme l'eau de source sans un traitement préalable. Ce phénomène est le plus remarqué à Adigbonou et Koudo où respectivement $84 \%$ et $82 \%$ de la population n'appliquent aucun traitement à l'eau avant la consommation tandis qu'une faible minorité (10\%) y ajoutent de l'aquatabs, de l'alun ou la porte à ébullition. De plus, l'observation sur le terrain a montré que la majorité de la population jettent des ordures et défèquent dans la nature. Des maladies hydriques comme la diarrhée et la dysenterie sont régulièrement enregistrées au sein des populations étudiées.

On a constaté que $100 \%$ des villages ne disposent pas de latrines. La majorité de la population jettent des ordures dans la nature et défèquent aux abords des cours d'eau.

Les données du $\mathrm{pH}$ sont comprises entre 4,77 et 6,55 et sont en dessous de la limite inférieure de la norme recommandée par l'OMS $(6,5<\mathrm{pH}<8,5)$ (Figure 2) ;

Toutes les eaux échantillonnées ont des températures situées entre $25^{\circ} \mathrm{C}$ et $28^{\circ} \mathrm{C}$.

Les conductivités obtenues se situent entre 20 et $60 \mu \mathrm{S} / \mathrm{cm}$.

La Figure 3 renseigne sur la couleur des eaux prélevées dans les villages. La couleur étant considérée comme l'un des paramètres les plus importants pour la qualité des eaux, elle est très élevée et dépasse largement la norme fixée par l'OMS qui est de 15 UC. Quant au fer total, les valeurs des échantillons des eaux dépassent la valeur maximale admissible qui est de 0.3 $\mathrm{mg} / \mathrm{l}$.

L'ammonium a une valeur maximale admissible de $0,5 \mathrm{mg} / \mathrm{l}$. Les valeurs obtenues pour les échantillons qui sont respectivement $1,986 \mathrm{mg} / \mathrm{l} ; 2,089 \mathrm{mg} / \mathrm{l} ; 1,380 \mathrm{mg} / 1 ; 1,393$ $\mathrm{mg} / \mathrm{l}$ dépassent la norme.

Les éléments azotés comme les nitrites et nitrates de toutes les eaux échantillonnées respectent la norme qui est de $0,1 \mathrm{mg} / \mathrm{l}$ pour les nitrites et de $50 \mathrm{mg} / \mathrm{l}$ pour les nitrates Les taux de nitrate obtenus varient de $0 \mathrm{mg} / 1$ à 14,52 $\mathrm{mg} / \mathrm{l}$. Par contre les eaux échantillonnées présentent des taux d'ammonium $\left(\mathrm{NH}_{4}{ }^{+}\right)$qui dépassent les valeurs maximales admissibles de $0,5 \mathrm{mg} / \mathrm{l}$. Les valeurs sont comprises entre 0,97 et $2,04 \mathrm{mg} / \mathrm{l}$. Seules les eaux prélevées dans les villages d'Adigbonou et de Koudou ont un taux d'ammonium très faible.

Quant à la Figure 5, les taux de calcium sont conformes à la norme fixée par l'OMS. Les concentrations varient de $0,8 \mathrm{mg} / \mathrm{l}$ à 8,2 $\mathrm{mg} / \mathrm{l}$ tandis que pour le magnésium elles varient de $0,49 \mathrm{mg} / \mathrm{l}$ à $4,38 \mathrm{mg} / \mathrm{l}$. Les plus faibles teneurs en calcium $(0,8 \mathrm{mg} / \mathrm{l})$ et en magnésium $(0,49 \mathrm{mg} / \mathrm{l})$ sont obtenues dans le village Koudo tandis que les valeurs élevées en calcium sont obtenues à Hlankpa $(8,02 \mathrm{mg} / \mathrm{l})$ et celles en magnésium respectivement à Gbanan et à Goukè. Quant au fer total, 100\% des eaux échantillonnées ont des concentrations en fer total au-delà de la norme $(0.3 \mathrm{mg} / \mathrm{l})$.

Le taux de chlorure varie de $10,65 \mathrm{mg} / \mathrm{l}$ à $320 \mathrm{mg} / \mathrm{l}$. La valeur la plus élevée est obtenue à Goutonou et dépasse la valeur recommandée par l'OMS qui est de $250 \mathrm{mg} / \mathrm{l}$.

Il ressort de la Figure 6 que les eaux prélevées ont une concentration en fluorures qui varie de 0 à $0,22 \mathrm{mg} / \mathrm{l}$. Toutes ces concentrations sont largement en dessous de la concentration maximale fixée par l'OMS qui est $1,5 \mathrm{mg} / \mathrm{l}$. l'eau prélevée à Goutonou n'en contient pas du tout.

Les analyses bactériologiques montrent à travers les Figures 7, 8, 9 et 10 que les eaux échantillonnées sont contaminées par des coliformes totaux, des coliformes fécaux et des streptocoques fécaux. Les valeurs obtenues sont supérieures à la norme bactériologique avec des valeurs allant de 150 à 22320 germes par $100 \mathrm{ml} \mathrm{d}$ 'eau pour les coliformes totaux, de 70 à 3200 germes par $100 \mathrm{ml}$ d'eau pour les coliformes fécaux et de 40 à 2000 germes pour les streptocoques fécaux alors que les normes recommandée par l'OMS pour une eau de boisson sont respectivement 0 UFC $/ 100 \mathrm{ml}$ pour les coliformes fécaux et les streptocoques fécaux et de 10 UFC $/ 100 \mathrm{ml}$ pour les coliformes totaux. La valeur la plus élevée de coliformes fécaux a été identifiée à Gbanan avec une concentration de $3200 \mathrm{UFC} / 100 \mathrm{ml}$. 


\section{pH, Température, conductivité (Adjohoun)}

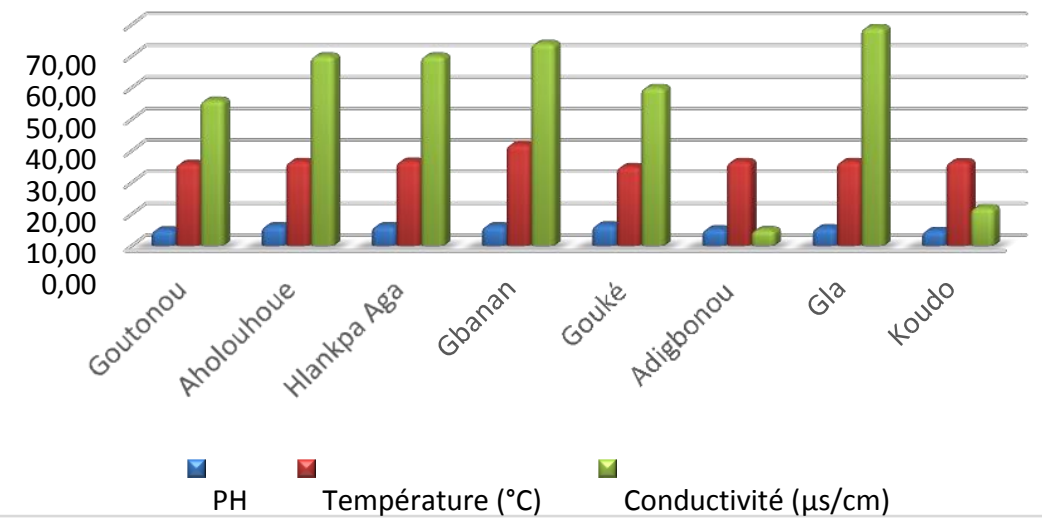

Figure 2: pH, Température et Conductivité.

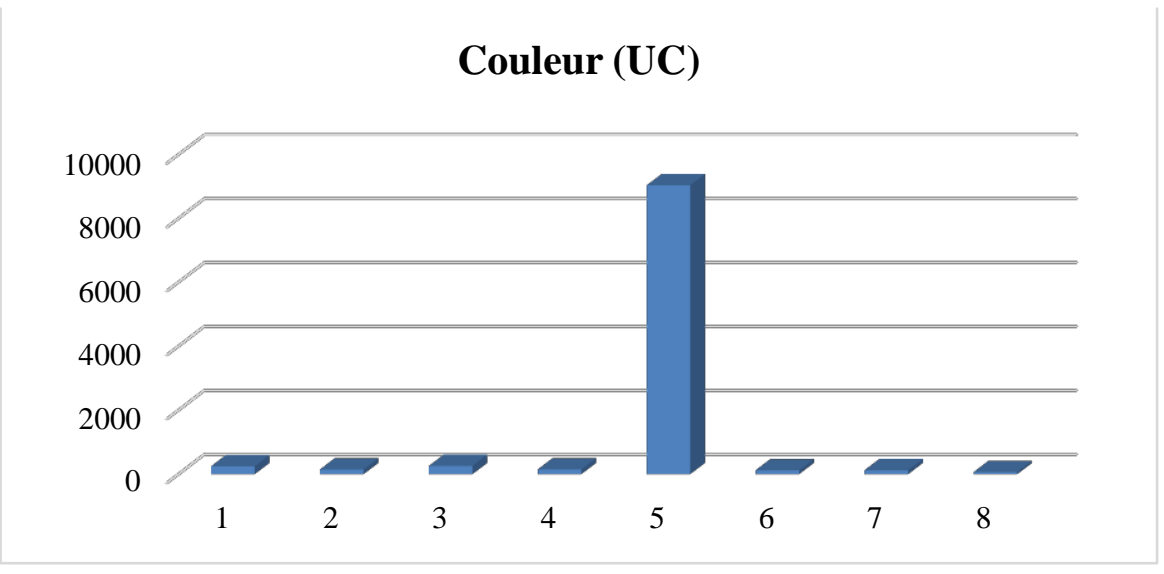

Figure 3: Couleur des eaux prélevées.

\section{Nitrite, Nitrate Ammonium (Adjohoun)}

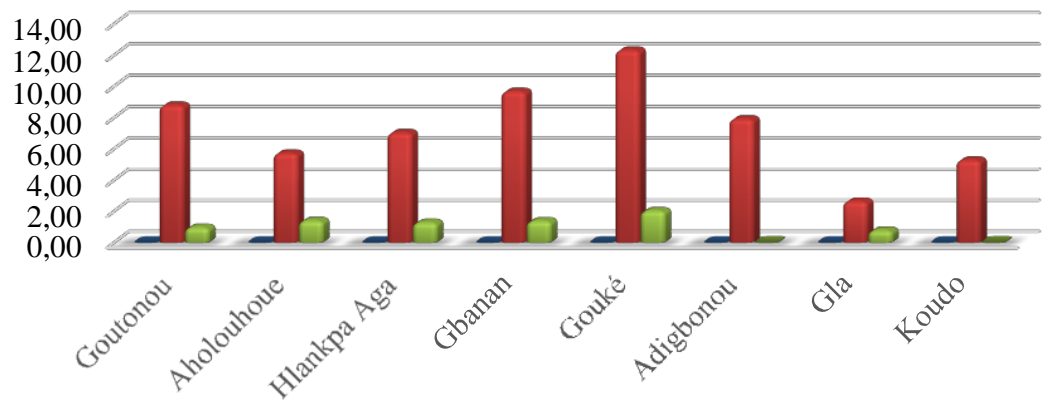

$\square$ Nitrite NO2- $(\mathrm{mg} / \mathrm{l}) \quad \square$ Nitrate NO3- $(\mathrm{mg} / \mathrm{l}) \quad \square$ Ammoniums NH4+ (mg/l)

Figure 4: Taux de nitrite, de nitrate et d'ammonium. 


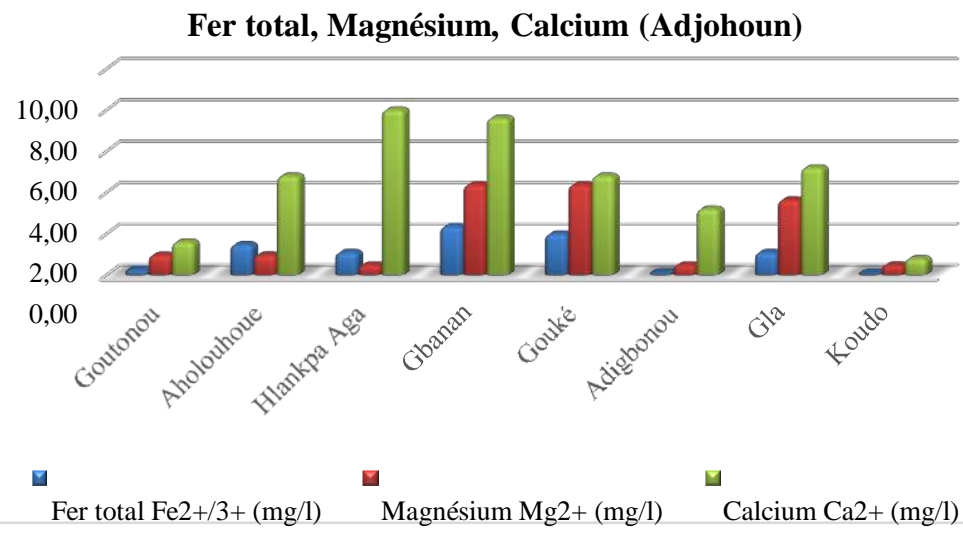

Figure 5: Taux de magnésium, de Calcium et de Ter total des eaux analysées.

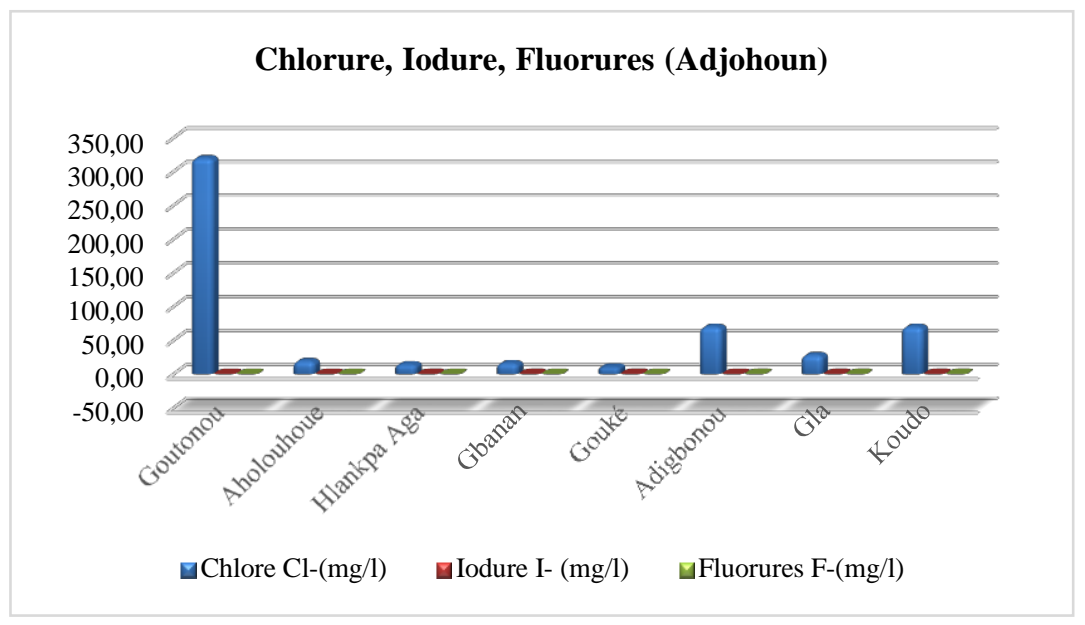

Figure 6: Taux de Chlorure, d'Iodure et de Fluorure.

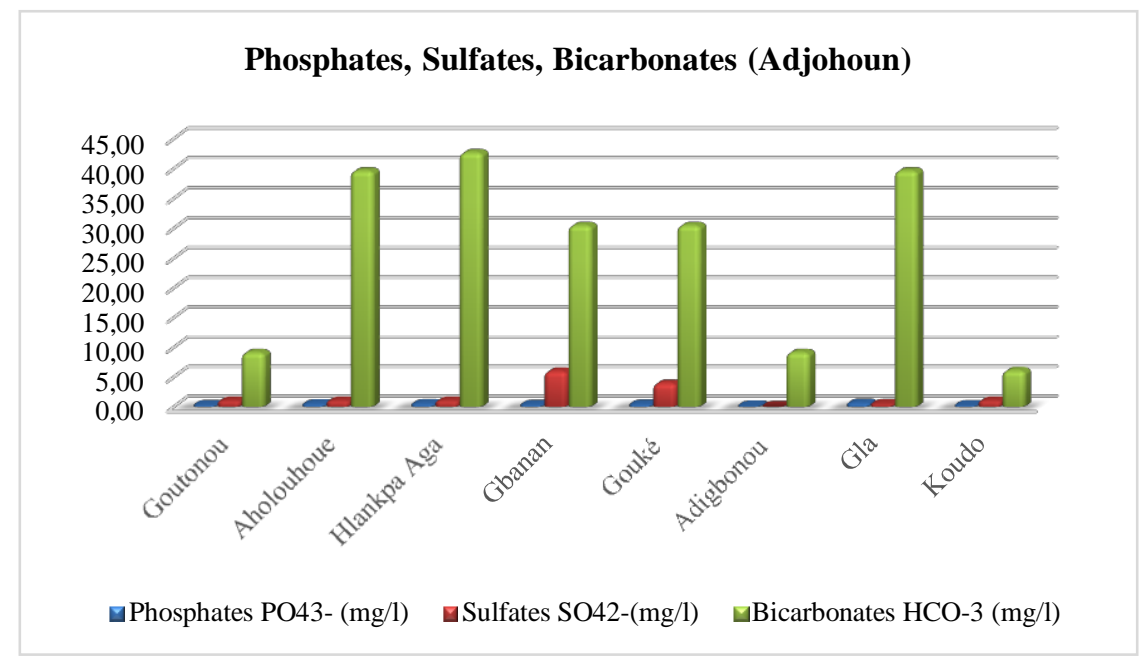

Figure 7: Taux de Phosphates, de Sulfates et de Bicarbonates. 


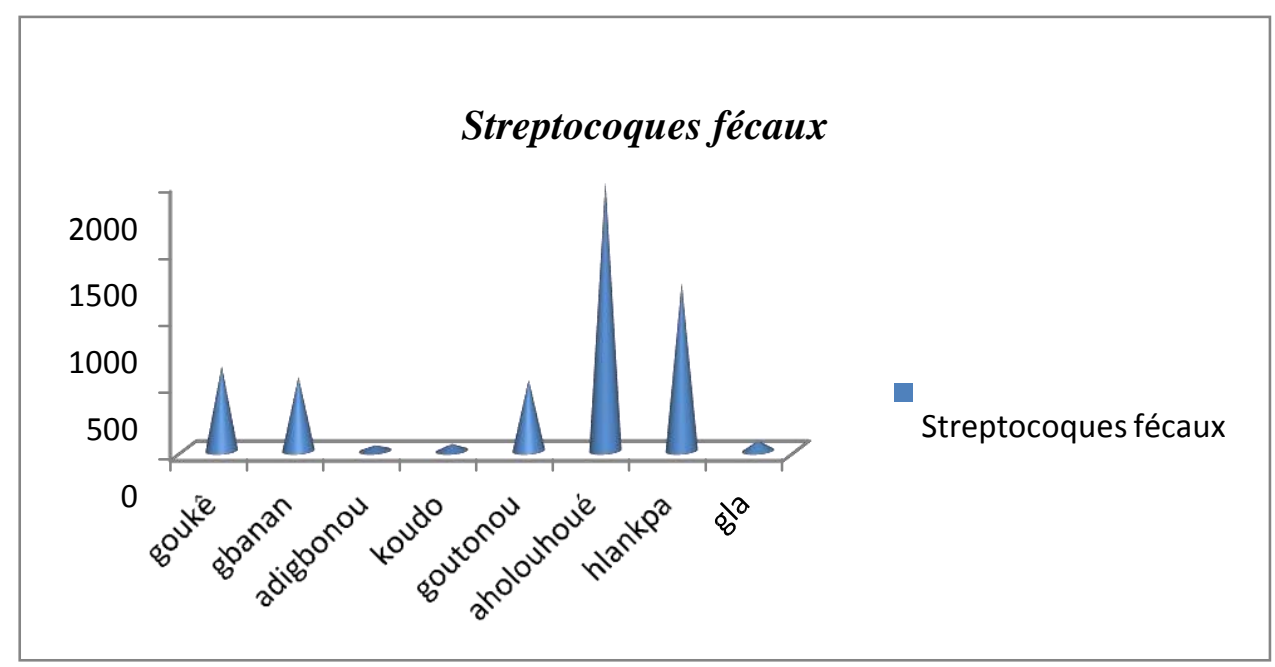

Figure 8: Streptocoques fécaux.

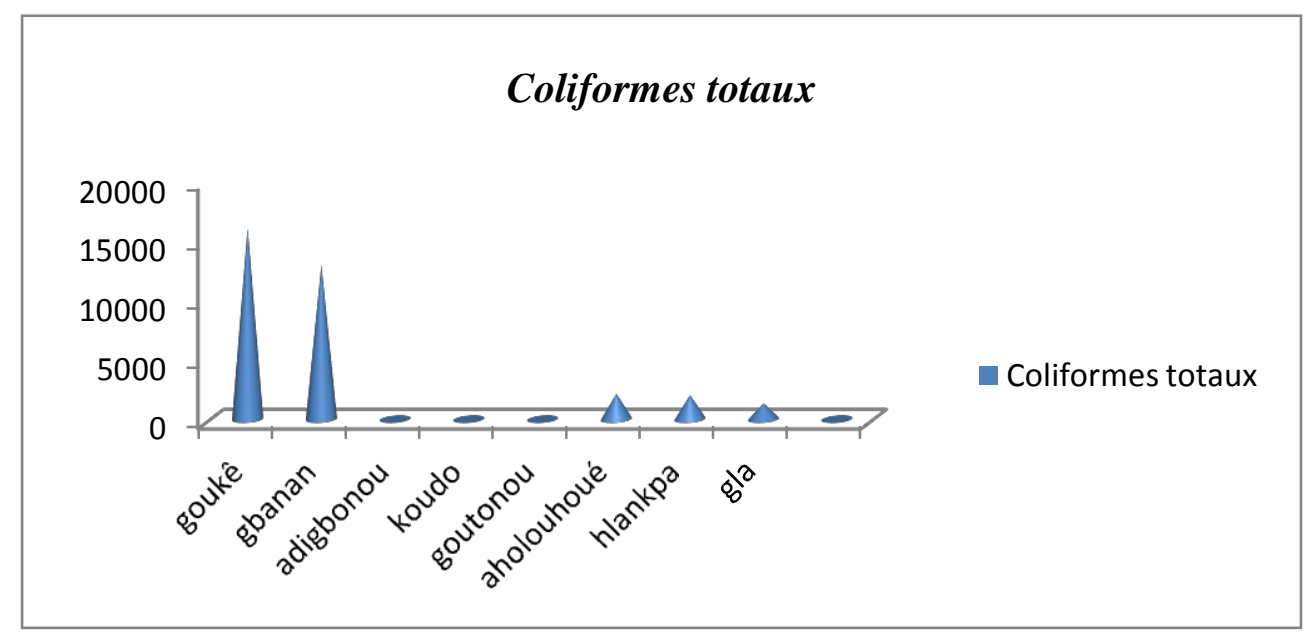

Figure 9: Coliformes totaux.

\section{Coliformes fécaux}

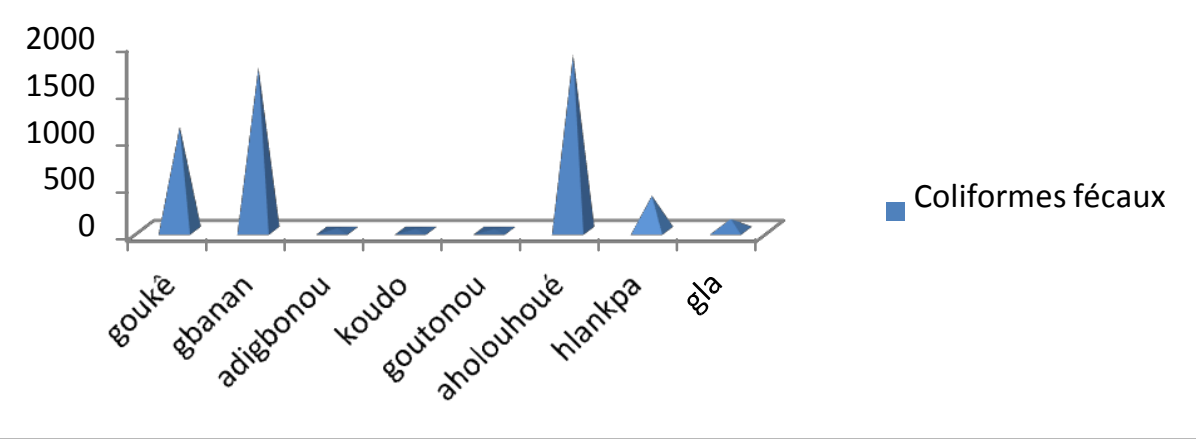

Figure 10: Coliformes fécaux. 


\section{DISCUSSION}

Des enquêtes ont été menées dans les différents villages identifiés afin de recenser les usages que les populations font de l'eau. La majeure partie de la population consomme l'eau de source sans un traitement préalable.

La Figure 2 montre que les eaux échantillonnées ont un $\mathrm{pH}$ légèrement acide. Le $\mathrm{pH}$ est un paramètre important de la qualité de l'eau. Il permet de définir le caractère agressif ou incrustant d'une eau. Les données obtenues sont comprises entre 4,77 et 6,55 et sont en dessous de la limite inférieure de la norme recommandée par l'OMS $(6,5<\mathrm{pH}<8,5)$ L'acidité de ces eaux proviendrait soit de la nature du sol, soit de la décomposition de la matière organique végétale. Quant à la température toutes les eaux échantillonnées ont des températures situées entre $25^{\circ} \mathrm{C}$ et $28^{\circ} \mathrm{C}$, constituent de bons milieux de culture pour les microorganismes de l'environnement comme l'a souligné Galal-Gortchev en 1992. Les conductivités obtenues se situent entre 20 et $60 \mu \mathrm{S} / \mathrm{cm}$. Selon l'OMS les eaux ayant des conductivités situées entre 100 et $200 \mu \mathrm{S} / \mathrm{cm}$ sont faiblement minéralisées et par conséquent sont des eaux douces. La Figure 3 renseigne sur la couleur des eaux prélevées dans les villages. La couleur étant considérée comme l'un des paramètres les plus importants pour la qualité des eaux, elle est très élevée et dépasse largement la norme fixée par l'OMS qui est de 15 UC. Par conséquent l'eau n'est pas propre. Cette grande augmentation pourrait être due soit à la nature du sol traversé, à la présence de fer, de manganèse de cuivre ou de matières organiques (Black et Christman 1963). Bien qu'une eau colorée ne comporte aucun danger pour la santé, la présence de couleur met mal à l'aise les consommateurs même si l'eau est de bonne qualité. (Dossa, 2013). Nos résultats sont similaires à ceux obtenus par Dossa qui a analysé les eaux de Ganvié, Ahomey-Glon et Ahomey-Ounmey en 2013. Il a obtenu une valeur élevée (148,5 UC) dans toutes les eaux échantillonnées et affirme que cette grande variation de la couleur n'est rien d'autre que la présence de matières organiques (substances humiques, fer, etc.). Quant au fer total, les valeurs des échantillons des eaux dépassent la valeur maximale admissible qui est de $0.3 \mathrm{mg} / \mathrm{l}$. Les résultats diffèrent de ceux obtenus par Edorh A.P et al 2010 qui a analysé les eaux de puits de Sèmé-Podji et a trouvé que 50\% des eaux analysées sont contaminées. La présence du fer dans l'eau donne une saveur désagréable, et un aspect rougeâtre à l'eau. Le fer est indispensable pour le corps humain mais de très fortes concentrations affectent les propriétés organoleptiques de l'eau, donne une saveur désagréable et un aspect rougeâtre à l'eau. Sa présence peut indiquer que l'eau est de mauvaise qualité.

L'ammonium a une valeur maximale admissible de $0,5 \mathrm{mg} / \mathrm{l}$. Les valeurs obtenues pour les échantillons dépassent la norme. Ces résultats sont similaires à ceux trouvés par Makoutode et al 2006 qui ont échantillonnés des eaux dans les communes d'Abomey-Calavi et de Grand Popo qui ont trouvé que $80 \%$ des eaux prélevées ont un taux supérieur en ammonium. La présence de l'ammonium dans l'eau, lui donne un goût désagréable et facilite le développement des germes.

Les éléments azotés comme les nitrites et nitrates de toutes les eaux échantillonnées respectent la norme qui est de $0,1 \mathrm{mg} / \mathrm{l}$ pour les nitrites et de $50 \mathrm{mg} / \mathrm{l}$ pour les nitrates. Nos résultats sont différents de ceux obtenus par Kotchadan en 2011 qui a analysé les eaux souterraines de la Commune de Dassa-Zoume et trouvé que $75 \%$ des eaux analysées ont un taux de nitrate supérieur à la norme. Par contre les eaux échantillonnées présentent des taux d'ammonium $\left(\mathrm{NH}_{4}{ }^{+}\right)$qui dépassent les valeurs maximales admissibles de $0,5 \mathrm{mg} / \mathrm{l}$. Seules les eaux prélevées dans les villages d'Adigbonou et de Koudou ont un taux d'ammonium très faible. Ces résultats sont similaires à ceux trouvés par Makoutode et al 2006 qui ont trouvé que $80 \%$ des eaux prélevées dans les communes d'Abomey-Calavi et de GrandPopo ont un taux supérieur en ammonium. La présence de l'ammonium dans l'eau, lui donne un goût désagréable et facilite le développement des germes. Les taux de calcium sont conformes à la norme fixée par l'OMS. Les plus faibles teneurs en calcium $(0,8$ $\mathrm{mg} / \mathrm{l})$ et en magnésium $(0,49 \mathrm{mg} / \mathrm{l})$ sont obtenues dans le village Koudo tandis que les 
valeurs élevées en calcium sont obtenues à Hlankpa $(8,02 \mathrm{mg} / \mathrm{l})$ et celles en magnésium respectivement à Gbanan et à Goukè. Quant au fer total, $100 \%$ des eaux échantillonnées ont des concentrations en fer total au-delà de la norme $(0.3 \mathrm{mg} / \mathrm{l})$. Le taux de chlorure le plus élevé est obtenu à Goutonou et dépasse la valeur recommandée par l'OMS qui est de 250 mg/l. Il ressort de la Figure 6 que les eaux prélevées ont une concentration en fluorures qui varie de 0 à $0,22 \mathrm{mg} / \mathrm{l}$. Toutes ces concentrations sont largement en dessous de la concentration maximale fixée par l'OMS qui est $1,5 \mathrm{mg} / \mathrm{l}$.

Les analyses bactériologiques montrent à travers les Figures 7, 8, 9 et 10 que les eaux échantillonnées sont contaminées par des coliformes totaux, des coliformes fécaux et des streptocoques fécaux. Les valeurs obtenues sont supérieures aux normes bactériologiques recommandée par l'OMS qui sont respectivement 0 UFC $/ 100 \mathrm{ml}$ pour les coliformes fécaux et les streptocoques fécaux et de 10 UFC /100 ml pour les coliformes totaux. Les fortes concentrations de coliformes totaux, fécaux et de streptocoques fécaux peuvent être attribuées aux divers déchets que reçoivent les cours d'eau, au manque d'hygiène et d'assainissement de l'environnement. Ces résultats sont en adéquation avec ceux de Dovonou (2012) qui a montré que la pollution du lac Nokoué est due au dépôt de branchages dans le lac, aux dépotoirs sauvages d'ordure implantés le long de la berge et à la défécation qui se fait directement dans les eaux. La présence des coliformes fécaux et des streptocoques fécaux indique une pollution strictement d'origine fécale et la présence probable d'autres germes ou parasites pathogènes qui peuvent être responsables des maladies. Pour Rodier (1984), ces forts taux exposent les populations à de nombreux risquent sanitaires.

\section{Conclusion}

Cette étude a permis d'avoir une idée de la qualité des eaux consommées par la population de la commune d'Adjohoun. Les travaux révèlent que sur le plan physicochimique certains paramètres comme la couleur, l'ammonium, le fer total ne respectent pas la norme de potabilité, ne sont pas conformes aux valeurs recommandées par les Directives de qualité de l'eau de boisson de l'OMS. Les analyses bactériologiques viennent confirmer quant à elle que les eaux analysées sont contaminées par des coliformes et des streptocoques qui seraient d'origine fécale. Cela pourrait s'expliquer par le manque d'hygiène et d'assainissement de l'environnement Les latrines étant absentes dans certains villages ils défèquent dans les cours d'eau. Les eaux consommées sont malsaines et constituent une source de maladie d'origine hydrique.

\section{CONFLIT D'INTÉRÊTS}

Les auteurs déclarent, à l'unanimité, qu'il n'y a aucun conflit d'intérêts qui serait liée au présent article.

\section{AUTHORS' CONTRIBUTIONS}

Tous les auteurs ont contribué à la réalisation de ce travail et à la rédaction du manuscrit.

\section{REFERENCES}

ASSITEB. 1997. Problématique de l'eau et de l'assainissement en milieu scolaire en République du Bénin: cas de la ville de Djougou. Mémoire de fin de stage, p.50.

Black A, Christman R. 1963. Characteristic of colored surface waters. J. Am. Water works Assoc., 55(6): 753-770. DOI: 10.1002/j.1551-8833.1963.tb01085.x 
Dovonou F. 2012. Pollution physico-chimique et bactériologique du lac Nokoué. Doctorat en hydrologie et gestion intégrée des ressources en eau, p.150.

Edorh AP, Yehouenou A, Pazou E, Agbangla C, Koumolou L, Honfonga RT, Amoussou CC. 2010. Qualité microbiologique et physicochimique de l'eau des puits côtiers : cas de Sèmé-Podji (BENIN). Climat et développement, 9: 8 .

Galal-Hortchev H. 1992. Evaluation des risques liés aux substances chimiques dans l'eau de boisson : élaboration des valeurs guides. Santé Publique, 4 : 80-83.

INSAE, RGPH3. 2003. Synthèses des analyses. p.48.

Kotchadan M. 2011. Paramètre physicochimiques et bactériologiques des eaux souterraines et des eaux de surface dans la commune de DASSA-ZOUME : Cas des villages Ayniwidji, Bétékoukou et Zankoumandon, p.46.

Makoutode DC, Ouendone FB, De Brouwer C. 2006. Qualité et mode de gestion de l'eau de puits en milieu rural du Bénin : cas de la sous-préfecture de GrandPopo.

http://www.jle.com/en/revues/santepub/e rs/edocs/00/04/3E/E9/article.phtml

(Consulté le 20/07/2014 à 11h20min).

Monographie de la Commune d'Adjohoun, Afrique Conseil 2006. p. 62. MSP. 2011.
Annuaire des statistiques sanitaires, Cotonou, $147 \mathrm{p}$.

OMS. 2006. Normes sur l'eau potable. www.lennetech.com/Français.fr.htm (Consulté le 20/07/2014 à 11h20min).

Plan de Développement Communal d'Adjohoun. 2011. p. 58.

Rodier J. 1976. L'analyse de l'eau, eaux naturelles, eaux résiduaires, eaux de mer. BORDAS. Paris-France, p.1383.

Rodier J. 1984. L'analyse de l'eau, eaux naturelles, eaux résiduaires, eaux de mer. DUNOD : France-Paris, p.1365.

UNICEF. 1998. Comment susciter un meilleur comportement en matière d'hygiène importance des mécanismes de changement en santé publique. La Haye, p.47.

Institut National de la Statistique. 2013. Qualité des eaux de surface et souterraines dans la ville de Yaoundé et son impact sanitaire. Une publication du département des Statistiques Démographiques et Sociales. Site web : www.statistics-cameroon.org (Consulté le 15 Août 2017 à 16H15).

http://www.cnrs.fr/cw/dossiers/doseau/decouv /potable.html

http://www.eaupotable.sante.gouv.fr ; http://www.observatoire-

environnement.org; www.google.fr; www.fr.wikipedia.org (Consulté le 7Août 2017 à 10h30). 